\title{
MicroRNA-221 accelerates the proliferation of laryngeal cancer cell line Hep-2 by suppressing Apaf-1
}

\author{
XIN SUN, BIN LIU, XU-DONG ZHAO, LI-YIN WANG and WEN-YUE JI \\ Department of Otorhinolaryngology, Shengjing Hospital, China Medical University, \\ Heping, Shenyang, Liaoning 110004, P.R. China
}

Received October 22, 2014; Accepted December 12, 2014

DOI: $10.3892 /$ or.2015.3714

\begin{abstract}
Laryngeal cancer is one of the most commonly occurring malignant cancers of the head and neck region. In the present study, we investigated the roles of miR-221 in laryngeal squamous cell carcinoma cell line, Hep-2. We examined the function and mechanism of miR-221 in Hep-2 cells using techniques of cell biology and molecular pathology, such as western blotting, quantitative PCR, immunohistochemical staining and flow cytometry. Using a luciferase assay, the apoptotic protease activating factor-1 (Apaf-1) mRNA 3'-UTR was shown to have complementary binding sites using bioinformatics prediction software including TargetScan, PicTar and miRanda. In conclusion, our results showed that miR-221 inhibition caused elevated expression levels of the Apaf-1 apoptotic pathway proteins caspase-3, -8 and -9 . miR-221 may therefore be used as a novel therapeutic target for laryngeal cancer.
\end{abstract}

\section{Introduction}

Laryngeal cancer is one of the most commonly occurring malignant cancers of the head and neck region (1). It rates second in incidence among head and neck cancers, and 11th among all human cancers (2). Advances in chemotherapy, radiation therapy and surgical techniques have improved the 5-year survival rate (2), however, recurrence is prevalent following treatment. Thus, identification of novel therapeutic targets for laryngeal cancer is crucial.

MicroRNAs (miRNAs) are short, non-coding RNA molecules that post-transcriptionally regulate the expression of target genes, and play a role in diverse cell, physiological and pathophysiological processes $(3,4)$. miR-221 is coded from chromosome $\mathrm{X}$ and functions as an oncogenic miRNA and is involved in various types of cancer (5). It was reported to be upregulated in many types of tumor, including glioblastoma,

Correspondence to: Dr Xin Sun, Department of Otorhinolaryngology, Shengjing Hospital, China Medical University, No. 36 Sanhao Street, Heping, Shenyang, Liaoning 110004, P.R. China E-mail: sunxin_sj@163.com

Key words: miR-221, Apaf-1, proliferation, apoptosis, survival rate bladder cancer and papillary tumors of the thyroid (6-8). miR-221 was shown to affect several cancer pathways by modulating multiple gene targets such as estrogen receptor- $\alpha$, p27, p57 and receptor tyrosine kinase kit 1 (c-kit) (9-12).

To the best of our knowledge, no previous study has shown the association between miR-221 and laryngeal squamous cell carcinoma (LSCC). The aim of the present study was to present the role of miR-221 in laryngeal cancer cell line, Hep-2. Furthermore, we focused on a target protein of miR-221, apoptotic protease activating factor-1 (Apaf-1). We also determined the function and underlying mechanism of miR-221 in Hep-2 cells.

\section{Materials and methods}

Cell culture and transfection. The human Hep-2 laryngeal cancer cell line was purchased from the Shanghai Institutes for Biological Sciences, Chinese Academy of Sciences. The cells were cultured in PRMI-1640 supplemented with $10 \%$ heatinactivated fetal bovine serum (FBS) (both from HyClone, Logan, UT, USA) in a humidified cell incubator with an atmosphere of $5 \% \mathrm{CO} 2$ at $37^{\circ} \mathrm{C}$. Exponentially growing cells were used for experiments. Hep-2 cells were transfected with miR-221 antisense and scrambled control oligonucleotides (Thermo Fisher, Waltham, MA USA) or Apaf-1 siRNA (sc-29201; Santa Cruz Biotechnology, Inc., Santa Cruz, CA, USA) using Lipofectamine 2000 (Invitrogen, Carlsbad, CA, USA) according to the manufacturer's instructions.

Bioinformatics analysis. The analysis of predicted miR-221 targets was performed using the algorithms TargetScan (http://targetscan.org/), PicTar (http://pictar.mdc-berlin.de/), and miRanda (http://www.microrna.org/microrna/home.do/) website tools. The minimum free energy predicted for hybridization was determined by BibiServ analysis (http://bibiserv. techfak.uni-bielefeld.de/genefisher2/).

Luciferase reporter assay. Hep-2 cells were co-transfected using Lipofectamine 2000 reagent (Invitrogen) with $100 \mathrm{ng}$ of firefly luciferase construct and $300 \mathrm{ng}$ of control-pcDNA3.1 or pcDNA3.1-miR-221 expression vector. A total of $10 \mathrm{ng}$ of pRL-CMV (Promega Biotech Co., Ltd., Beijing, China) was co-transfected as a normalization control. Reporter assays were performed $48 \mathrm{~h}$ post-transfection using the Dual-Luciferase 
Assay system (Promega, Madison, WI, USA), normalized for transfection efficiency by the co-transfected Renilla luciferase.

MTT assay. Cells were plated in 96-well plates at a concentration of 1,500 cells/well and allowed to attach overnight. The 3-(4,5-dimethylthiazol-2-yl)-2,5-diphenyltetrazolium bromide (MTT) solution (Sigma-Aldrich, Carlsbad, CA, USA) was then added at a final concentration of $0.5 \mathrm{mg} / \mathrm{ml}$ for $4 \mathrm{~h}$. After $4 \mathrm{~h}$, the cells were lysed with dimethyl sulfoxide (DMSO; Sigma) and absorbance rates were measured at $550-560 \mathrm{~nm}$ using a microplate reader (Bio-Rad, Hercules, CA, USA).

Apoptosis analysis. As per the manufacturer's instructions for the Apoptosis Assay kit (KeyGen Nanjing, China), the stained cells were analyzed by flow cytometry (BD Biosciences, Rockville, MD, USA). Data analysis was performed using CellQuest software (BD Biosciences).

Cell cycle. The cells were collected and fixed in $70 \%$ ethanol at $4^{\circ} \mathrm{C}$ for $16 \mathrm{~h}$. Fixed cells were then washed once with phosphate-buffered saline (PBS), resuspended in $500 \mathrm{ml}$ PBS containing $10 \mu \mathrm{g} / \mathrm{ml}$ propidium iodide (KeyGen) and $50 \mathrm{mg} / \mathrm{ml} \mathrm{RNase}$ and incubated for $30 \mathrm{~min}$ at room temperature. The cells were then centrifuged at 1,200 rpm for $5 \mathrm{~min}$, resuspended in $500 \mathrm{ml}$ of PBS and analyzed with FCM.

Xenograft assays. Animal studies were performed according to the institutional guidelines. Hep-2 $\left(3 \times 10^{7}\right.$ in $\left.200 \mu \mathrm{l}\right)$ cells were injected subcutaneously into the right flanks of 6-8 weeks male nude mice (Charles River, Wilmington, MA, USA). Treatment was initiated after the tumor diameters reached 3-5 $\mathrm{mm}$ by daily intratumoral injections of PBS, miR-221 antisense or scrambled control oligonucleotides or Apaf-1 siRNA for two cycles of 3 days. Tumor growth was then monitored for 30 days. Every five days until the end of the experiment, one mouse from each group was randomly selected to be anesthetized, photographed and sacrificed. The tumor volumes were determined by measuring the length (l) and the width (w) and calculating the volume $\left(\mathrm{V}=\mathrm{lw}^{2} / 2\right)$. Tumor samples were analyzed by western blotting. Additional mice $(n=60)$ were used to establish xenografts to obtain survival curves. Mice with xenografted tumors (as described above) that reached 3-5 $\mathrm{mm}$ in diameter were divided into three treatment groups $(\mathrm{n}=20$ for each). Survival was monitored until the experiments were terminated due to the heavy tumor burden.

In situ hybridization of $m i R$-221. A tissue array containing 36 murine tissue samples from the groups described above was created using a tissue microarrayer MIA-I (Beecher Instruments, Silver Spring, MD, USA). In situ hybridization (ISH) was carried out with double-digoxigenin (DIG)-labeled miRCURY LNA ${ }^{\mathrm{TM}}$ miR detection probe against has-miR-221 (Exiqon, Copenhagen, Denmark) according to the supplier's instructions.

Quantitative PCR analysis of $m i R-221$. After thawing at $4^{\circ} \mathrm{C}$, $400 \mu 1$ of cyst fluid was subjected to small RNA extraction using the mirVana ${ }^{\mathrm{TM}}$ miRNA isolation kit (Ambion, Applied Biosystems, Austin, TX, USA) according to the manufacturer's instructions. Quantitative PCR (qPCR) was analyzed using the
Bulge-Loop ${ }^{\mathrm{TM}}$ miRNA RT-qPCR Detection kit (Ribobio Co., Guangzhou, China) and TransStart ${ }^{\mathrm{TM}}$ Green qPCR SuperMix (TransGen Biotech, Beijing, China) according to the manufacturer's instructions with the Rotor-gene 6000 system (Qiagen, Hilden, Germany). The reactions were incubated at $95^{\circ} \mathrm{C}$ for $30 \mathrm{sec}$, followed by $40 \mathrm{cycles}$ of $95^{\circ} \mathrm{C}$ for $30 \mathrm{sec}, 60^{\circ} \mathrm{C}$ for $20 \mathrm{sec}$, and $70^{\circ} \mathrm{C}$ for $1 \mathrm{sec}$. The relative expression level for miRNA221 was computed using the comparative $\mathrm{Ct}$ method. miRNA expression was normalized to small nucleolar RNA U6.

Western blotting. Tissues were rinsed twice with cold PBS buffer and lysed in an ice-cold lysis buffer containing $150 \mathrm{mM}$ $\mathrm{NaCl}, 50 \mathrm{mM}$ Tris- $\mathrm{HCl}$ (pH 7.6), 0.1\% SDS, $1 \%$ Nonidet P-40, and a protease inhibitor cocktail (Roche, Basel, Switzerland). The extracts were incubated on ice for $20 \mathrm{~min}$, centrifuged at $12,000 \mathrm{xg}$ for $20 \mathrm{~min}$ at $4^{\circ} \mathrm{C}$, and the supernatants were collected. Protein concentrations were determined using Bradford assay (Bio-Rad), and proteins were resolved by $10 \%$ Bis-Tris gel electrophoresis, transferred to a nitrocellulose membrane, and western blot analysis was performed. Rabbit polyclonal IgG anti-Apaf-1 (sc-8339), mouse monoclonal IgG anti-caspase-3 (sc-7272), mouse monoclonal IgG anti-caspase-8 (sc-56070), mouse monoclonal IgG anti-caspase-9 (sc-73548) and mouse monoclonal IgG anti- $\beta$-actin (sc-47778) were purchased from Santa Cruz Biotechnology, Inc. (Santa Cruz).

Immunohistochemical staining. Immunohistochemical (IHC) staining was performed on $4-\mu \mathrm{m}$ sections obtained from formalin-fixed, paraffin-embedded blocks. Endogenous peroxidase activity was blocked with $3 \%$ hydrogen peroxide for $30 \mathrm{~min}$. Antigen retrieval was carried out in citrate buffer $\left(10 \mathrm{mM}, \mathrm{pH}\right.$ 6.0) for $30 \mathrm{~min}$ at $95^{\circ} \mathrm{C}$ in a microwave oven. The sections were incubated with primary antibody as described in western blotting at $4^{\circ} \mathrm{C}$ overnight. The sections were then incubated with a biotinylated secondary antibody and then exposed to a streptavidin complex (HRP; Sigma). Positive reactions were visualized with 3,3'-diaminobenzidine (DAB) tetrahydrochloride, followed by counterstaining with hematoxylin (both from Sigma).

Statistical analysis. Statistical analyses were carried out using GraphPad Prism version 5.00 for Windows (GraphPad Software, San Diego, CA, USA). Numerical data were presented as means $\pm \mathrm{SD}$. The Student's t-test and one-way ANOVA analysis was used to determine significance. Kaplan-Meier survival plots were generated and comparisons between survival curves were made with the log-rank statistic. $\mathrm{P}<0.05$ was considered to indicate a statistically significant result. The experiments were conducted in triplicate.

\section{Results}

miR-221 interacts specifically with the 3'UTR region of Apaf- 1 . First of all, we identified Apaf- 1 as the potential target of miR-221 using TargetScan and miRanda online search programs. A $100 \%$ matched sequence was found at the 154-160 nucleotide region of Apaf-1 mRNA 3'UTR (Fig. 1A). The free energy was calculated as $\sim-11.4 \mathrm{kcal} / \mathrm{mol}$ for the hybrid of the Apaf-1 3'UTR region and miR-221 by the Bibiserv analysis (Fig. 1A). Secondly, we confirmed the hypothesis that 

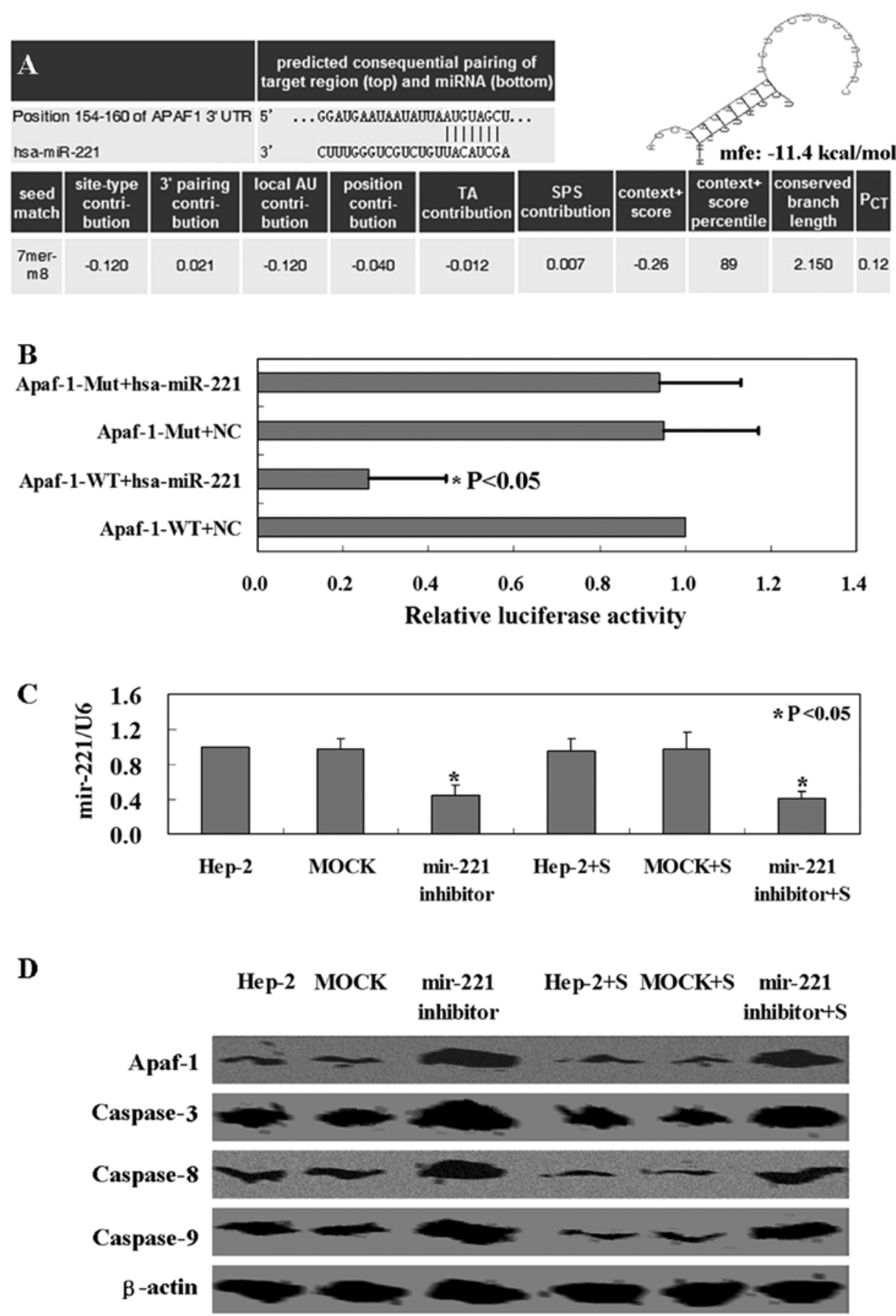

Figure 1. Apaf-1 is a target of miR-221. (A) Image of the predicted miR-221-binding sequences in the 3'UTR region of Apaf-1. The calculated free energy for hybridization of Apaf-1 and miR-221. (B) Luciferase assay of pRL3-Apaf-1 and anti-miR-221 in Hep-2 cells. (C) The levels of miR-221 were measured using quantitative PCR. U6 was used as an internal control. (D) Western blot analysis was performed to detect apoptosis-related proteins. Cell lysates were electrophoresed and Apaf-1, caspase-3, -8 and -9 proteins were detected by their specific antibodies. $\beta$-actin was used as an internal control. Hep-2, untreated Hep-2 cells; MOCK, Hep-2 cells transfected with scrambled control oligonucleotides; miR-221 inhibitor, Hep-2 cells transfected with miR-221 antisense; Hep-2+S, Hep-2 cells transfected with Apaf-1 siRNA; MOCK+S, Hep-2 cells transfected with scrambled control oligonucleotides and Apaf-1 siRNA; miR-221 inhibitor+S, Hep-2 cells transfected with miR-221 antisense and Apaf-1 siRNA. Apaf-1, apoptotic protease activating factor-1.

miR-221 targets the 3'UTR region of Apaf-1 using a luciferase reporter assay. Co-transfection of miR-221 expression vector along with the full-length 3'UTR of Apaf-1 caused a significant decrease in luciferase units compared to the controls $(\mathrm{p}<0.05$, Fig. 1B). Furthermore, we decreased miR-221 expression in Hep-2 cells using miR-221 antisense ( $p<0.05$, Fig. 1C). Compared with mock-transfected or untransfected ones, Apaf-1 protein in Hep-2 cells was markedly increased after
miR-221 antisense transfection $(\mathrm{p}<0.05$, Fig. 1D). The results clearly demonstrated that miR-221 targeted specifically the 3'UTR region of Apaf-1.

Effect of miR-221 and Apaf-1 on Hep-2 cells in vitro and in vivo. The MTT assay showed that the proliferation rate of Hep-2 cells with miR-221 antisense transfection was decreased compared to the untransfected or mock-transfected 

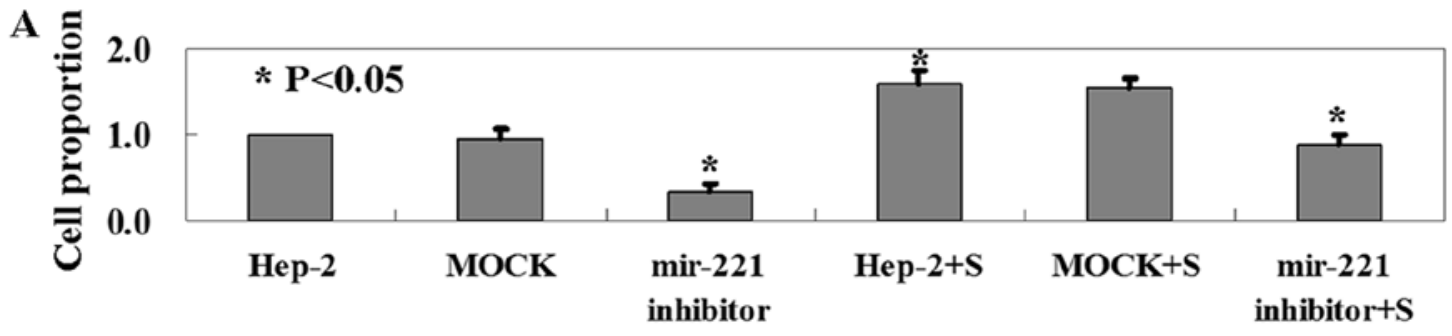

B
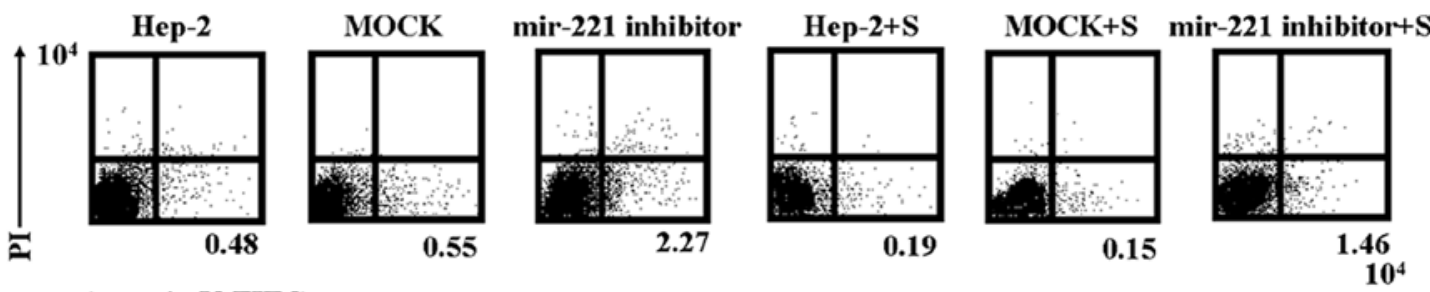

Annexin V-FITC
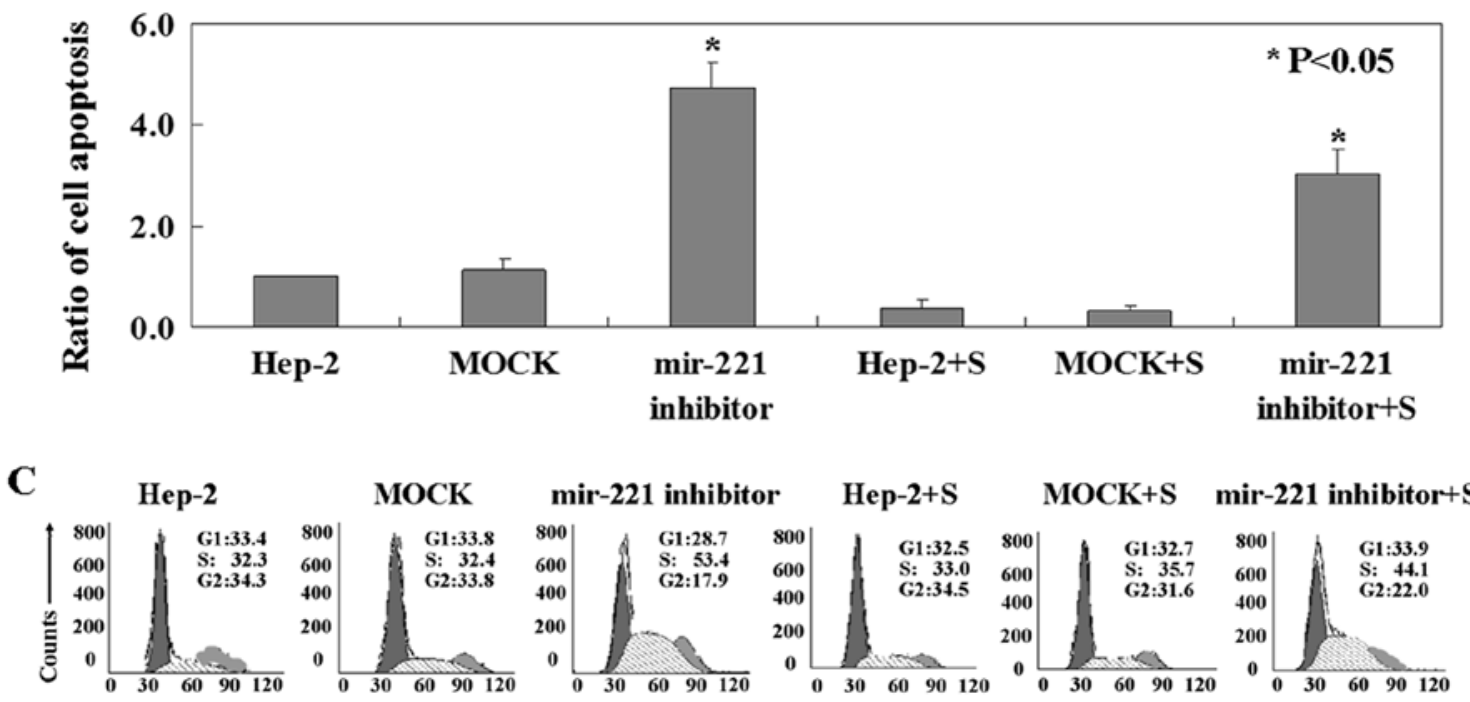

Figure 2. The effects of miR-221 inhibition on Hep-2 cells. (A) The percentages of growth inhibition of the downregulation of miR-221 or Apaf-1 on Hep-2 cells were determined by MTT assay in comparison with the untreated ones. (B) Apoptotic ratio of Hep-2 cells was analyzed by double staining with Annexin V/propidium iodide (PI). (C) The cell cycle was determined using PI staining. Apaf-1, apoptotic protease activating factor-1.

cells ( $<<0.05$, Fig. 2A). The cells with Apaf-1 knockdown showed a higher proliferation rate than the untransfected or mock-transfected ones (p<0.05, Fig. 2A). As shown in Fig. 2B, the ratio of apoptotic Hep-2 cells with miR-221 antisense transfection was 5- to 6-fold higher than that in the untransfected or mock-transfected ones $(\mathrm{p}<0.05)$. Apaf-1 knockdown cells exhibited a lower apoptotic ratio than other cells ( $\mathrm{p}<0.05$, Fig. 2B). As evidenced by PI staining, miR-221 antisense caused a significant increase in the fraction of S-phase cells (Fig. 2C). Apaf-1 knockdown partly repaired miR-221-induced S-phase arrest and promoted cell cycle restoration (Fig. 2C).

In vivo efficacy of miR-221 and Apaf-1 in a human Hep-2 xenograft mouse model. The success of intratumoral injection of miR-221 antisense was demonstrated using qPCR and ISH, respectively. The results of qPCR $(p<0.05$, Fig. 3D) and ISH (Fig. 3E) showed a lower level of miR-221 in the tumor tissues of the mice transfected with miR-221 antisense as compared to other groups. The tumor volume and weights of the mice transfected with miR-221 antisense were lower than those of the PBS or mock group. However, the tumor volume and weights of the Apaf-1 knockdown group was higher than the other groups ( $\mathrm{p}<0.05$, Fig. $3 \mathrm{~A}$ and B). Correspondingly, the survival rate of mice with miR-221 antisense was significantly improved, while the Apaf-1 knockdown group showed a poor survival rate ( $\mathrm{p}<0.05$, Fig. $3 \mathrm{C})$.

Mechanism of miR-221 in Hep-2 cells. To determine the mechanisms of miR-221, western blotting and IHC were carried out to measure the changes of possible proteins. The level of Apaf-1 protein was observed to be higher in Hep-2 cells with miR-221 antisense transfection compared with untreated cells (Fig. 1D). Expression levels of caspase-3, -8 and -9 were also higher and associated with the Apaf-1 expression (Fig. 1D). The same mechanism of miR-221 was confirmed using the tissues of Hep-2 xenograft mouse model (Fig. 3E). The results suggested that miR-221 inhibits Apaf-1 expression in Hep-2 cells, followed with the downregulation of caspase- $-3,-8$ and -9 . 
A

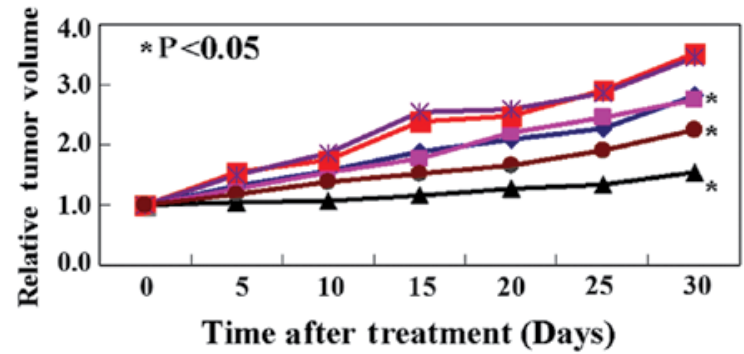

B

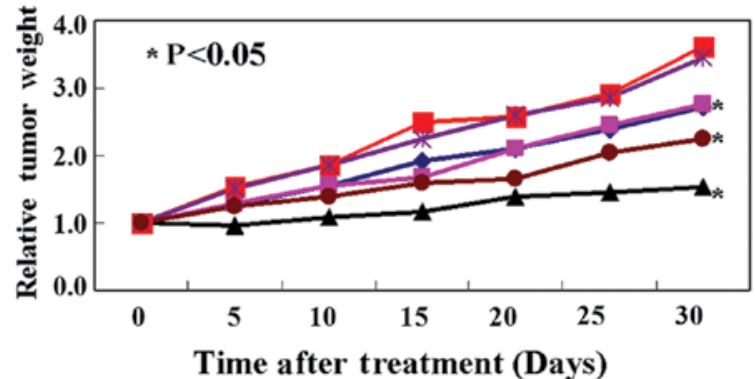

C

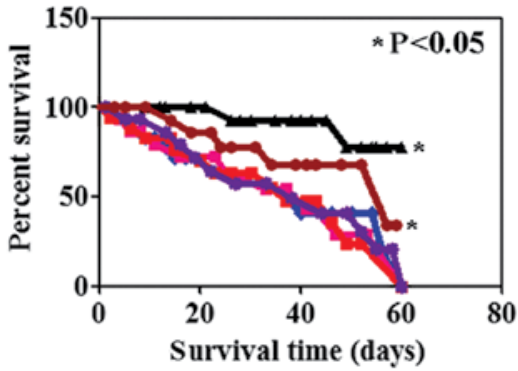

$\rightarrow$ Hep-2

- MOCK

- mir-221 inhibitor

- Hep-2+S

- MOCK+S

- mir-221 inhibitor+S $\multimap$ Hep-2

- - MOCK

士 mir-221 inhibitor

- -Hep-2+S

$\rightarrow-\mathrm{MOCK}+\mathrm{S}$

$\multimap$ mir-221 inhibitor+S

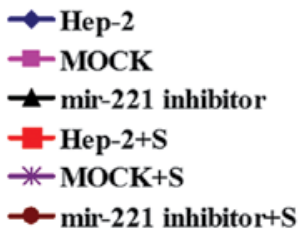

$\rightarrow$ Hep-2

$=$ MOCK

$\triangle$ mir-221 inhibitor

- - Hep-2+S

* MOCK $+\mathrm{S}$

$\rightarrow-$ mir-221 inhibitor $+S$

D

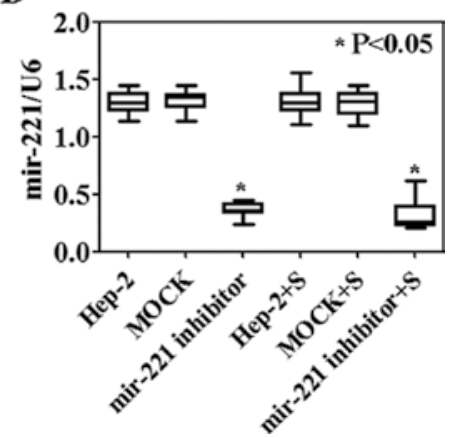

E

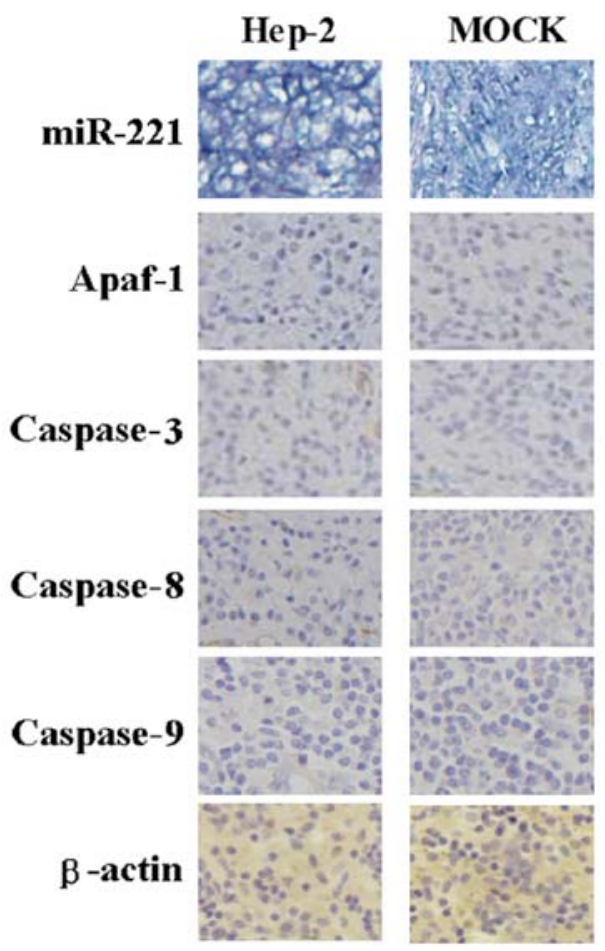

mir-221
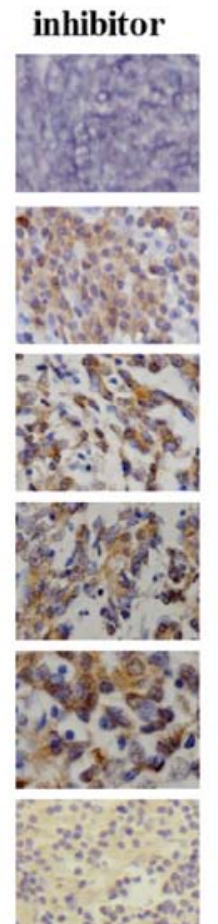
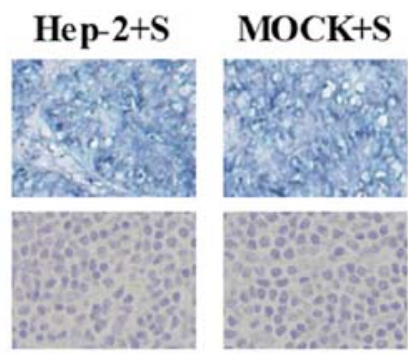

mir-221

inhibitor $+\mathrm{S}$
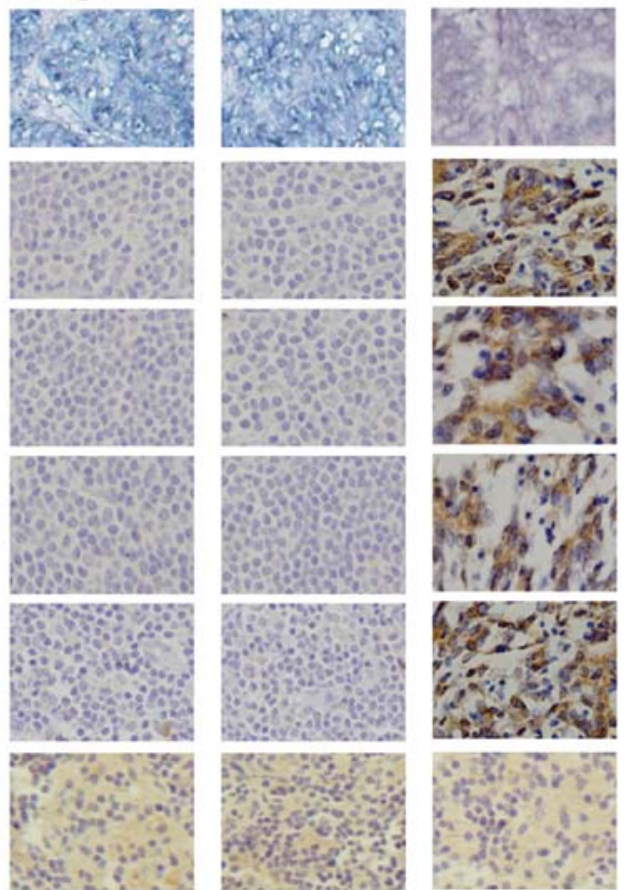

Figure 3. miR-221 inhibition suppressed tumor growth in LSCC xenograft mouse models. (A and B) Tumor volume and weights of the groups described in Materials and methods. (C) Kaplan-Meier survival curves of the groups described in the Materials and methods. (D) The levels of miR-221 were measured using quantitative PCR. U6 was used as an internal control. (E) miR-221 expression in tumor specimens from xenograft mouse models was detected by in situ hybridization. Apaf-1, caspase- $3,-8$ and -9 proteins were detected by immunohistochemical staining. Bound antibodies were detected with DAB and appear brown. LSCC, laryngeal squamous cell carcinoma; Apaf-1, apoptotic protease activating factor-1. 


\section{Discussion}

The results of the present study have demonstrated that inhibition of miR-221 in Hep-2 cells induced apoptosis in vitro and in vivo. miR-221 is upregulated in multiple malignancies (6-8). Overexpression of miR-221 promoted cancer cell proliferation by its ability to inhibit the expression of the cyclin-dependent kinase inhibitors CDKN1B/p27 $(13,14)$. We also confirmed that miR-221 inhibition suppressed cell proliferation and induced apoptosis in Hep-2 cells. Previous studies have shown that miR-221 promotes tumor cells entering the $S$ phase from the $\mathrm{G}_{1}$ phase $(15,16)$. In the present study, the downregulation of miR-221 was identified to induce S-phase arrest. Park et al (17) found that anti-miR-221 treatment improved survival of the orthotopic tumor xenograft mice compared with the scrambled control. Consistent with that study, we found miR-221 inhibition decreased tumor volume and weights, although the survival rate of Hep-2 xenograft mouse models was improved.

Furthermore, we found that miR-221 and Apaf-1 mRNA 3'-UTR have complementary binding sites using bioinformatics prediction software including TargetScan, PicTar and miRanda. However, whether Apaf- 1 is a novel target of miR-221 in LSCC has yet to be reported. The hypothesis was demonstrated using the luciferase assay. The results of the luciferase assay showed a direct interaction between miR-221 and the target site in the 3'-UTR of the Apaf-1 mRNA. In addition, we confirmed that a decreased miR-221 level was associated with the upregulation of Apaf-1 expression in vitro and in vivo. The results collectively confirmed Apaf-1 as a novel target of miR-221.

Apaf-1 is a component protein of the apoptosome (18). Apoptosis was induced by exogenous and endogenous factors (19). In the intrinsic apoptotic pathway, cytochrome $c$, Apaf-1 and caspase-9 precursor are interacted, and then caspase-9 precursor becomes activated (20). Apaf-1 downregulation has been connection with decreased apoptosis and correlated with adverse prognosis in colorectal cancer (21). Overexpression of Apaf-1 in the U87 cells has an effect on inhibiting cell proliferation and promoting apoptosis (22). Consistent with previous studies, our results also revealed Apaf-1 expression induced Hep-2 cell apoptosis and cell cycle arrest. Furthermore, results of the present study showed that downregulation of miR-221 caused elevated expression levels of the Apaf-1 apoptotic pathway proteins caspase-3, -8 and -9 .

In conclusion, the present study reports the knockdown of endogenous miR-221-inhibited proliferation, induced apoptosis and cell cycle arrest of laryngeal cancer cell. miR-221 was demonstrated to possess apoptosis resistance function in laryngeal cancer cell by targeting Apaf-1. Of note is that we confirmed the antitumor roles of miR-221 in animal models. miR-221 may therefore be employed as a novel therapeutic target of laryngeal cancer.

\section{Acknowledgements}

This study was supported by the Doctoral Scientific Start-up Funds of Liaoning Province (no. 20141041).

\section{References}

1. Chen YF, Luo RZ, Li Y, et al: High expression levels of COX-2 and P300 are associated with unfavorable survival in laryngeal squamous cell carcinoma. Eur Arch Otorhinolaryngol 270: 1009-1017, 2013

2. Chu EA and Kim YJ: Laryngeal cancer: diagnosis and preoperative work-up. Otolaryngol Clin North Am 41: 673-695, 2008.

3. Chang TC and Mendell JT: microRNAs in vertebrate physiology and human disease. Annu Rev Genomics Hum Genet 8: 215-239, 2007.

4. Latronico MV, Catalucci D and Condorelli G: Emerging role of microRNAs in cardiovascular biology. Circ Res 101: 1225-1236, 2007.

5. Ergun S, Arman K, Temiz E, et al: Expression patterns of miR-221 and its target caspase-3 in different cancer cell lines. Mol Biol Rep 41: 5877-5881, 2014.

6. Ciafrè SA, Galardi S, Mangiola A, et al: Extensive modulation of a set of microRNAs in primary glioblastoma. Biochem Biophys Res Commun 334: 1351-1358, 2005.

7. Gottardo F, Liu CG, Ferracin M, et al: MicroRNA profiling in kidney and bladder cancers. Urol Oncol 25: 387-392, 2007.

8. He H, Jazdzewski K, Li W, et al: The role of microRNA genes in papillary thyroid carcinoma. Proc Natl Acad Sci USA 102: 19075-19080, 2005.

9. Medina R, Zaidi SK, Liu CG, et al: MicroRNAs 221 and 222 bypass quiescence and compromise cell survival. Cancer Res 68 : 2773-2780, 2008.

10. le Sage C, Nagel R, Egan DA, et al: Regulation of the $\mathrm{p} 27^{\mathrm{Kipl}}$ tumor suppressor by miR-221 and miR-222 promotes cancer cell proliferation. EMBO J 26: 3699-3708, 2007.

11. Gramantieri L, Fornari F, Ferracin M, et al: MicroRNA-221 targets Bmf in hepatocellular carcinoma and correlates with tumor multifocality. Clin Cancer Res 15: 5073-5081, 2009.

12. Fu X, Wang Q, Chen J, et al: Clinical significance of miR-221 and its inverse correlation with $\mathrm{p} 27^{\mathrm{Kipl}}$ in hepatocellular carcinoma. Mol Biol Rep 38: 3029-3035, 2011.

13. Visone R, Russo L, Pallante P, et al: MicroRNAs (miR)-221 and miR-222, both overexpressed in human thyroid papillary carcinomas, regulate $\mathrm{p} 27^{\mathrm{Kipl}}$ protein levels and cell cycle. Endocr Relat Cancer 14: 791-798, 2007.

14. Gillies JK and Lorimer IA: Regulation of $\mathrm{p} 27^{\mathrm{Kip} 1}$ by miRNA 221/222 in glioblastoma. Cell Cycle 6: 2005-2009, 2007.

15. Ahmed FE, Jeffries CD, Vos PW, et al: Diagnostic microRNA markers for screening sporadic human colon cancer and active ulcerative colitis in stool and tissue. Cancer Genomics Proteomics 6: 281-295, 2009.

16. Felli N, Fontana L, Pelosi E, et al: MicroRNAs 221 and 222 inhibit normal erythropoiesis and erythroleukemic cell growth via kit receptor down-modulation. Proc Natl Acad Sci USA 102: 18081-18086, 2005.

17. Park JK, Kogure T, Nuovo GJ, et al: miR-221 silencing blocks hepatocellular carcinoma and promotes survival. Cancer Res 71: 7608-7616, 2011

18. Li P, Nijhawan D, Budihardjo I, et al: Cytochrome c and dATPdependent formation of Apaf-1/caspase-9 complex initiates an apoptotic protease cascade. Cell 91: 479-489, 1997.

19. Lian S, Shi R, Bai T, et al: Anti-miRNA-23a oligonucleotide suppresses glioma cells growth by targeting apoptotic proteaseactivating factor-1. Curr Pharm Des 19: 6382-6389, 2013.

20. Zang YS, Zhong YF, Fang Z, et al: MiR-155 inhibits the sensitivity of lung cancer cells to cisplatin via negative regulation of Apaf-1 expression. Cancer Gene Ther 19: 773-778, 2012.

21. Paik SS, Jang KS, Song YS, et al: Reduced expression of Apaf-1 in colorectal adenocarcinoma correlates with tumor progression and aggressive phenotype. Ann Surg Oncol 14: 3453-3459, 2007.

22. Chen L, Zhang J, Han L, et al: Downregulation of miR-221/222 sensitizes glioma cells to temozolomide by regulating apoptosis independently of p53 status. Oncol Rep 27: 854-860, 2012. 